\title{
Magie de l'horreur: Qui se souvient de la mer de Mohammed Dib
}

\author{
Lucia Trifu \\ Queen's University
}

D ans son étude ${ }^{1}$ consacrée aux créations littéraires maghrébines qui traitent de la guerre d'indépendance de l'Algérie, Jean Déjeux énumère près de trente-deux romans, sans compter les nouvelles ou les poèmes. Chose remarquable, de cette riche production, s'inscrivant dans la lignée des oeuvres littéraires d'expression française, Qui se souvient de la mer de Mohammed Dib s'impose comme le premier grand roman sur l'immensurable tragédie de ce peuple. Les enthymèmes, visant la représentation de l'horreur en art, qui constituent la postface du livre, ont conduit l'auteur à explorer des moyens artistiques insolites: ceux-ci, selon Dib, se rapprochent du modèle créé par Picasso dans Guernica, même si le contexte historique, la tradition culturelle et esthétique sont totalement différents pour les deux artistes.

La question que l'écrivain maghrébin soulève est celle de savoir comment il est possible de transformer un tel événement en une émotion esthétique durable car il y a du possible à dire et de l'indicible. Autrement dit, il s'agit de considérer les moyens à mettre en oeuvre pour présenter des choses aussi terrifiantes, lesquelles inspirent l'horreur et la crainte. Et ce, après Auschwitz et Hiroshima qui fonctionnent comme des baromètres de la souffrance humaine, comme des "modèles abominables de l'incommensurabilité"2? L'Histoire est pleine de "noms sans nom" et il faut toujours les reconsidérer, les relier, pour que cela ne puisse se reproduire jamais. Si Picasso a inventé une construction "inhumaine" où se dit 1'horreur de la guerre civile d'Espagne, Dib aussi opère une rupture, subvertit les codes de la représentation et crée un langage inédit qui donne à ses visions une force nouvelle. La toile de Picasso, on le sait, n'est pas une représentation traditionnelle de bataille car pour le peintre l'art est artifice, technique, virtuosité, interprétation et invention de la réalité. Chef de file du cubisme, mouvement qui s'institue dans une critique de l'art mimétique, de cette tenace chimère selon quoi l'art copierait le réel, Picasso écarte de sa peinture tout détail réaliste, taches de sang, héroïsme, et recherche des motifs personnels de signification capables d'exprimer l'intensité de ses sentiments à l'égard de ce fait historique. Cela ne 
veut pas dire que la violence est voilée ou transformée: elle s'identifie à la destruction même, à la désintégration; elle est provoquée par les moyens mêmes de sa représentation. Ainsi Guernica n'est pas une toile historique, elle est le portrait mythique de l'histoire: un Guernica intemporel qui stigmatise le drame de la guerre et ses horreurs. Arnheim, un des critiques de l'oeuvre picassienne, mentionnait à propos de la célèbre toile que les "déformations de l'espace et les bizarreries du sujet sont aussi réalistes qu'une reproduction photographique, mais à un niveau de réalité différent"3. Ses affirmations trouvent leur pleine pertinence puisque la vérité n'est pas dans l'effet d'exactitude ni dans l'imitation parfaite mais dans la saisie de chaque aspect de la réalité avec sa valeur intrinsèque. Guernica est la grande révélation prospective d'autres cataclysmes tout aussi affreux, le symbole des victimes de la guerre en tout lieu, car l'illustre Espagnol transgresse l'événement particulier de l'histoire de son peuple et l'élève à l'échelle de l'universalité.

Il s'agira donc, dans mon étude, de considérer les techniques scripturales que Dib transpose à partir de ce que Picasso a fait dans sa peinture, au plan du texte et de la représentation narrative, de rechercher les modalités de l'écrivain pour inscrire ce qui n'est pas scriptible et dépasse l'entedement. La réalité plus invraisemblable que la fiction, pousse l'écrivain algérien à s'en éloigner à son tour, et à opter pour un autre monde, celui des fantasmes, fait qui suppose la déconstruction et la destruction de l'autre concret. L'auteur refuse de la sorte l'optique réaliste et nous propose en échange un espace tout aussi réel, mais qui constitue le monde sensible des éléments impalpables, des émotions, du lyrisme, de l'imaginaire. Au lieu d'une "reconstitution historique" il préfère "un cadre terrible et légendaire" puisqu'aucune horreur ne résiste à la répétition, aucune violence à l'excès de sa projection. Le temps plonge tout dans l'usure. Le problème qui se pose pour les deux artistes est donc de chercher à élaborer les moyens de lui échapper, à partir du moment où l'on est conscient de ses effets nuisibles. Une écriture de science-fiction, d'anticipation, de pressentiment traversée d'interventions surnaturelles, imprégnée de mythes "anciens et modernes les plus actifs" nous introduit dans un univers apocalyptique et semble offrir à Dib la meilleure formule pour "nommer ce qui n'a strictement pas de nom". Même si la rencontre de Qui se souvient de la mer avec la littérature de science - fiction est fortuite, comme l'écrivain l'affirme dans son méta-texte, elle est significative et ne peut pas être passée sous silence.

A la manière des surréalistes qui visaient la création d'un mythe collectif, incarnation de la fusion totale de l'imaginaire et du réel, du monde objectif et subjectif, Dib crée un monde symbolique, qui ne fait qu'un avec le monde réel. Si l'écrivain, à l'instar de Picasso et des surréalistes ${ }^{5}$, se tourne vers une autre réalité, "plus vitale", transcendante, on le sait ce n'est pas un acte gratuit, il le fait déterminé par plusieurs raisons qu'on essaiera d'analyser. Ricoeur montre que les mythes ne sont pas des histoires fausses, irréelles; ils "explorent sur un mode imaginatif notre rapport au monde, aux êtres, à l'être" . L'importance accordée au mythe s'explique par le rôle déterminant du mythe dans l'existence de l'humanité: il inscrit la volonté de survivre et de résister à toute forme de sujétion. De cette façon, le mythe constitue un prétexte pour sauver l'existence humaine 
de la nature fragmentaire du temps, la préserver de son caractère éphémère et dérisoire, en lui assurant une valeur et une dignité. Parole située hors de la temporalité, le mythe s'inscrit dans le temps historique pour lui donner un nouvel éclairage: "En faisant de l'histoire et de son modèle intemporel des reflets réciproques, [...] l'histoire mythique [...] représente une tactique d'annulation de l'historique, d'amortissement de l'événementiel"7. Certes, l'utilisation de la mythologie pourrait traduire l'importance accordée au passé, mais à la fois celle-ci, par la mythologisation même des faits historiques, des événements, imprime un caractère relativisant à l'histoire en tant que catégorie épistémologique. D'emblée, elle sera liée à fingere, c'est-à-dire à l'invention, à la fiction. Qui se souvient de la mer s'institue ainsi en représentation valide d'un passé mutilé, oublié, enterré par l'autre.

Dans le monde de l'aliénation, la vie quotidienne se déroule dans une atmosphère perpétuelle d'inquiétude et de catastrophe imminente, menaçante à chaque instant. L'étouffement qu'elle impose, les paniques, les révoltes rendent l'événement de la guerre inexplicable et inexprimable. C'est justement par les mythes, sorte de révélateurs comme en photographie, que les choses se dévoilent, se démasquent; le monde devient parlant, dénudé. Frantz Fanon indique un autre enjeu de l'écrivain indigène qui remplace l'affrontement objectif avec le colon par un affrontement mythique:

L'atmosphère de mythe et de magie, en me faisant peur, se comporte comme une réalité indubitable [...]. Les forces du colon sont infiniment rapetissées, frappées d'extranéité. On n'a plus vraiment à lutter contre elles puisque aussi bien ce qui compte c'est l'effrayante adversité des structures mythiques ${ }^{8}$.

En fait, l'écrivain, en intégrant la pensée magique dans son discours narratif, tente d'imprimer la marque d'une résistance et ainsi on comprend mieux le rôle subversif latent du mythe.

Après avoir fait ce rapide survol visant à identifier quelques aspects du fonctionnement des mythes dans le roman, on tentera de déterminer s'il y a parenté entre les mythes de Picasso et ceux de Dib. Si Picasso a su par son art transmuter la réalité contemporaine en mythes qu'il a adaptés aux nécessités de sa peinture, mettant en harmonie les mythes et ses motifs personnels de signification, Dib, à son tour, invente des mythes modernes, adéquats aux exigences de son écriture fantastique. Ainsi, le Minotaure, le mythe essentiel de Picasso, sera repris également par l'écrivain. Ferrier' suggère, à l'égard du Minotaure de Picasso, d'y voir non seulement l'incarnation de la violence, le symbole du déchaînement incontrôlable de la force aveugle, mais aussi une analogie entre l'espace labyrinthique qu'il occupe et l'espace hétérogène du tableau, composante qui se retrouve dans le texte de l'auteur maghrébin aussi et sur laquelle on reviendra dans cette étude car elle exige une explication. Pour l'instant notons que Dib, en considérant les traits physiques et les attributs de violence, de brutalité du Minotaure picassien, opère une translation afin de symboliser dans son monstre l'image de l'Autre. Il n'est plus, comme chez le peintre de Guernica, proximité tensionnelle des oxymorons, un taureau au visage semi-humain; cette fois le 
Minotaure est l'humain devenu bête féroce - créature étrange, "lance-flammes en avant" (10) dont les simples mouvements provoquent des sifflements - le minotaure de Dib est présence fantomatique, spectrale, grise, vaguement définie. Il serait important de signaler ici que nulle part dans le roman de Dib, l'occupation française n'est désignée clairement. Toujours nommée à la dérobée, toujours entourée d'un halo mystérieux qui attire et repousse à la fois:

Des minotaures veillent aux carrefours: à vrai dire des momies qui ont été ressucitées et affectées à ce service. De leur sommeil millénaire, beaucoup gardent encore une immobilité, une rigidité, dont elles ont quelque mal à se débarasser. Cela ajouté à leur regard de lézard, elles inspirent une terreur salutaire qui se traduit par un grand respect (46).

Représentants des autorités coloniales, les minotaures se montrent insensibles et implacables car enfermés dans leur "blindage", rien ne les touche. "La bête, sorte de fauve sombre" (98), "les errants qui pénètrent partout: dans les magasins, les habitations et prennent possession de la place qui leur convient" (67) sont toujours des images de l'autre. Aussi imprécises sont les données concernant les techniques de guerre de ces créatures évasivement définies. Il est difficile d'identifier les machines sophistiqées désignées par des termes tout neufs, inventés: "les spyrovirs rapides et doux" (102) ou les iriaces, "ces oiseaux guerriers inaccessibles à l'amitié" (106), qui crient et s'agitent au milieu des flammes avec lesquelles ils se confondent (33). Et la "théorie du comportement des iriaces", que l'écrivain élabore en étudiant "leur direction, le nombre, les heures de leurs sorties et de leurs retraites" (133) ne sert-elle pas à lire, comme naguère les oracles, ce qui va arriver? De même, les nouvelles constructions adressent "un avertissement" qu'il faut comprendre (146); ce sont là autant d'éléments qui font signe et annoncent l'ébranlement de ce monde et la naissance d'un autre, nouveau. C'est d'ici que découle le caractère de "vision et pressentiment" (189) de l'écriture dibienne, fait qui permet de saisir le but de l'auteur: encadrer la réalité de l'actualité, tout en nous proposant un développement dans le futur. Cela renvoie à Breton, chez qui le mythe est également conçu comme un moyen concret de liberté et comme une projection de l'avenir. "Le type de la metabkha", "l'ingénieur rigoureux" qui ajuste "la machine [...] avec la superbe aisance d'un cyclotron" (25) est l'annonciateur de ce futur qui se prépare dans les entrailles de la terre; l'avenir est placé ainsi sous l'emprise du présent puisque c'est à partir des possibilités qui constituent le "maintenant" que se figure ce futur. A partir des signes de prémonition, c'est un mythe de la création qui se dessine, ce qui équivaut à un bouleversement radical:

Un ancien et silencieux cataclysme nous ayant arrachés à nous-même et au monde, seul un nouveau cataclysme pouvait nous y reprojeter. Mais jusque-là, nous allions poursuivre notre existence selon l'ordre qui nous avait été légué, le destin désaffecté, refroidi, vidé de sa puissance, échoué au fond de chacun de nous. Jusque-là, le temps 
passerait, et quoi qu'il advînt, rien n'arriverait. Il y avait bien une maison, un domaine, des tâches à mener, mais tout cela n'était qu'un mensonge. Nous promenant dans cette simulation de la réalité, nous traversions bien plus vivantes que nous, les choses, auxquelles nous n'offrions pas la moindre résistance (78).

L'image du désastre absolu, nous laisse déceler la colonisation avec son virtuel ordre immuable qui s'enferme dans l'intervalle de mensonge. Le second cataclysme, qui est du même ordre que le premier, mais en sens contraire, vise la guerre de libération de l'Algérie. Dib va encore plus loin, en imaginant une perturbation à l'échelle de l'univers. Sa prémonition extrêmement perspicace de la débâcle mondiale postcoloniale se matérialise ainsi dans un mythe inédit de la création. Mais avant de parvenir à la naissance du monde nouveau, ou pour mieux dire à la re-naissance du monde, car il s'agit non d'une "naissance habituelle mais plutôt de la répétition de la naissance archétypale"10, on éprouve un stade obligatoire de métamorphoses, c'est-à-dire une dégradation successive et à la fois, une stratégie politique du fait qu'elle implique la question de formes de résistance adaptées à une situation tragique, intenable. Ce concept clé chez les surréalistes, la métamorphose, mystérieux "passage" - transformation d'un corps en un autre, création des monstres, est reprise à la mythologie où elle est chose courante.

On change d'aspect, de visage pour que la différence s'efface dans d'"informes moellons" (86), on est transformé en ruisseau de sang (148), on vit dans la peur d'être transformé en quelque monstre qui est justement l'adversaire de l'immortalité humaine. Le plus souvent, on est changé en pierre. En partant des attributs de la pierre: inflexibilité, dureté, rigidité, insensibilité, Dib trace les contours de la vie tragique, inhumaine des Algériens et de ses marques inscrites comme des tatouages. Ce passage vers l'anorganique, vers le minéral, la désagrégation que le souffle de la vie subit fait penser à une transformation de l'horreur en silence et c'est contre lui que l'écrivain prend la parole. De la sorte, les visages, topoi par excellence de l'expression des sentiments, traduisant l'angoisse causée par la guerre, deviennent des paysages arides, inhabités, visages géographiques ou paysages faciaux, des silhouettes difformes, enlaidies par l'exacerbation de la douleur, tout comme chez Picasso où la physionomie devient métaphore plastique: les dents, la langue sont cri hystérique, l'oeil n'est plus oeil, mais il est larme.

L'autre oeil, l'oeil-soleil de Guernica, centre irradiant de toute la douleur renvoie à un autre générateur de métamorphoses, emblème de semi-mort dans le roman de Dib. Le signe solaire, l'astre néfaste auquel, selon les termes de Ferrier, Icare vient se brûler les ailes est assimilé d'habitude dans la mythologie égyptienne au souverain, voire au colon chez Dib (28) car il se lie aux autres composantes destructrices de la vie sous l'oppression coloniale, rendant "l'air dur et sec", plongeant "la terre privée d'ombre" dans "la torride amertume" (32).

Rattachée au mythe du souverain, du fait de son autorité, la symbolique du père est significative dans la littérature maghrébine par son lien avec la quête identitaire. Dib remet en question le mythe du père, et lui en substitue un autre, celui de la mer-mère-femme, 
bouleversant ainsi les coordonnées de l'ordre de la pensée indigène puisqu'en Islam elle est oubliée. L'image-femme véhiculée par Dib, n'est plus médium préféré de l'expérimentation des tourments physiques et émotionnels, de la souffrance extrême comme chez le peintre; elle est celle de la continuité, de la tranquillité, celle qui assure la permanence de la vie. Symbolisée par la mer, elle incarne la source de la vie et de l'énergie créative. Se souvenir d'elle, c'est se souvenir de la mère, femme-mère des enfants, de celle "qui n'abandonne pas [...]"11. On sait que la mère est lieu sacré par excellence, celui où le mystère de la vie est caché, mais la mer-mère redouble ce rôle; en tant que gardienne de l'ancienne tradition, "répétition infinie des mêmes rites", des valeurs spirituelles, elle sera celle qui les transmettra à ses enfants. Ainsi, elle est non seulement matrice créatrice mais aussi formatrice. Espoir, promesse de l'avenir, elle maintient la vie et aide l'homme à se relever par un ressourcement profond dans "la grotte" inépuisable de l'origine.

On a déjà mentionné l'analogie évoquée par Ferrier entre l'espace labyrinthique du Minotaure et l'espace hétérogène de la toile picassienne, où formes humaines et bêtes, jour et nuit, dedans et dehors, vie et mort s'immiscent subrepticement. Il semble que le roman de Dib par ses entorses narratives à la chronologie de l'histoire, par la succession affective des événements, par le déploiement analytique de l'espace à l'instar des peintre cubistes, nous conduit vers une logique pareille de la pensée. Dès lors le rôle de Nafissa dont le nom en arabe signifie "respiration", "souffle de la vie", "âme", celle qui guide l'homme et lui montre la voie dans le dédale de la guerre, ne serait-il similaire à celui d'Ariane qui donna à Thésée venu en Crête pour combattre le Minotaure, le fil à l'aide duquel il pu sortir du labyrinthe? Cela parait d'autant plus pertinent que la mer-mère-femme est présente dans le titre du roman de Dib et revient tout au long du récit, s'imposant comme une continuité, comme le fil conducteur et unificateur des fragments épars de la narration. Dans ce contexte, en essayant de motiver le conflit dans l'ordre du texte, autrement dit, ce genre d'écriture fragmentée, éclatée, Khatibi montre que les bouleversements entre pays, civilisations, langues, provoqués par une violence historique, forment un traumatisme qui va se graver dans la mémoire du texte en tant "qu'inscriptions conflictuelles"12. Dérèglement de l'histoire, dérèglement du fil narratif qui nous suggère l'inachevé.

Dans des moments de crise comme celui de la guerre, on a vu comment l'écriture des mythes et de magie peut offrir une explication et une justification de l'histoire. Parallèlement à la désintégration du temps, Dib procède au brouillage de l'autre dimension déterminante de notre existence: 1'espace. La ville est générique, sans nom, car pour l'auteur algérien la guerre est coma temporel et spatial. Dès le début, quand nous sommes introduits dans la metabkha "au plafond bas et enfumé, aux murs tachés de graisse" (13), on nous dit que l'endroit n'a pas d'importance: en tant que témoin unique d'un espace qui déborde le cadre algérien, l'auteur témoigne pour le monde entier. Il est vrai, on peut trouver des noms géographiques réels: Ouahrane, Tafrata, Remchi, etc., et à ceux-ci s'ajoutent quelques mots arabes qui nous donnent la spécificité locale, mais le manque de détails est intentionnel, car le dessein de Dib est justement de perturber les coordonnées

\section{LittéRéalité 34}


spatio-temporelles.

En fait, toute l'action du livre, s'il y en a une, est construite à partir des déplacements réels ou statiques entre plusieurs niveaux d'espace. Ainsi, l'espace urbain, reflet des structures sociales imposées par la communauté dominante où les éléments social et spatial sont étroitement imbriqués, engendre maintenant, à cause de la guerre, plusieurs niveaux: l'ancienne ville, ou la ville de l'air et la ville souterraine. A la surface, la cité bâtie par "les autres", par les forces d'occupation:

La disposition de la ville: un enchevêtrement de boyaux creusés dans le basalte sur plusieurs étages, avec quelques fentes sur l'air libre mais qu'il est fort difficile de repérer, cette disposition même facilite la communication. En effet, sommes-nous plus qu'un dédale à l'intérieur d'un autre dédale à présent, abstraction faite de la faculté que nous avons de nous déplacer? (24).

Espace de la ville des nouvelles constructions et espace de la ville souterraine entre lesquels l'être est tiraillé, car en réalité, la guerre se révèle, dans le roman de Dib, comme une rupture et une oscillation au lieu de la dichotomie. Excroissance abominable en béton et verre dans le territoire-corps de la ville ancienne, l'espace de la ville étrangère est celui qui dédifférencie le tissu de la medina, striant le paysage lisse du nomade qui lui sert à présent de "moyen de communication"13. Il a aussi une autre connotation car il est endroit de rencontre avec l'altérité, de contact douloureux et lieu de piège: "Est-ce là que gît le secret de cette attirance que de nombreux habitants ont payé de leur vie?" (97). La ville étrangère agit donc comme par magie: par l'aspect merveilleux, par le mensonge, par le leurre, elle captive et séduit l'habitant indigène en le plaçant dans un écart par rapport à sa "propre" cité, en le déviant de sa direction de déplacement. Matérialisation durable de l'oppression de l'indigène, les nouvelles constructions ont été érigées par le sacrifice, qu'il faut entendre selon les termes derridiens comme la "mise à mort de l'unique en ce qu'il a d'unique, d'irremplaçable et de plus précieux"14 — de toute la population — "des enfants, des femmes en font les frais" (61).

L'on a ainsi un espace urbain à deux composantes: enfer ou espace des damnés, espace du présent, de l'air, restrictif, multiple contre le paradis, abri dans la terre inépuisable et suprême, espace continu du passé qui engendre aussi l'embryon de l'avenir. Surgit ici un élément inédit: car Dib opère, dans son roman, un renversement des deux termes: paradis et enfer, par rapport à l'eschatologie traditionnelle; l'enfer est en haut, alors que le paradis, espace de l'action clandestine est en profondeur ${ }^{15}$. Dans son postcommentaire au roman, Dib suggère le mariage de ces deux espaces: جنَّة و جهنَ (djanna wa djahannam) union de l'espace féminin (en arabe) du paradis et celui masculin de l'enfer: c'est à cette association que lui semblent appartenir les choses. L'idée d'une fusion entre les deux villes apparaît comme l'unique justification ou comme une possible compensation du malheur qu' on doit éprouver.

La violence de la guerre déborde, excède, elle est puissance démesurée, exorbitante par 
définition, échappant à toute limitation et restriction. Tout l'espace bouge et nul frein ne s'impose pour tempérer l'atmosphère brûlante qui brise le tympan et brouille la rétine. La réalité torrentielle où les murs immobiles remuent comme soumis à des spasmes, à des sortilèges, la violence des contrastes, le dynamisme des images, le tempo syncopé et les sensations brutales désarticulent l'être, l'arrachent à lui-même. Déshérité, sans avoir une place, l'Algérien "flotte (99), est "suspendu" (102), saisi par la lévitation. Les gens sont poussés "hors d'eux mêmes" (66) comme si le corps devenait inapte à contenir une souffrance et une humiliation débordantes. Carcasse matérielle indissociable de l'être, le corps est siège des phénomènes étranges provoqués par la déchirure: dédoublement (46), transsubstantiation $(27,168)$, réinvention de l'apparence (77). Le coeur, organe vital par excellence, "lieu du trésor invisible et indescellable"16 abandonne le corps et part (39) puisqu'on est écoeuré par l'angoisse et qu'on vit au seuil. Genre d'existence végétative, latente, métissage entre la vie et la mort.

La vie est vécue comme un cauchemar, le rêve inséré dans la vie: pour surmonter et traverser les ténèbres de l'anxiété inspirées par une réalité sordide. Dans un monde vide, le sommeil n'est plus un recours, ni un repos, il devient cauchemar du sang (33) où l'espace à parcourir se dilate davantage, rendant tout encore plus effrayant. L'espace est ainsi lieu où tout peut arriver, même l'inimaginable.

Le temps échappe à la chronologie, l'espace en dérive, insituable, devient à son tour lieu déconcertant de l'irréalité dans laquelle Dib nous introduit. Le langage, le seul qui permet de surprendre cette folie motrice universelle, subit, lui aussi, une déconstruction. Dib nous prévient, dans le méta-texte ajouté à son roman quant aux enjeux, instituer une distance par rapport au modèle traditionnel du roman réaliste et créer un autre modèle esthétique, analogue à celui que propose la peinture du célèbre Espagnol. Il s'agira donc, maintenant, de cerner d'autres moyens artistiques que l'écrivain algérien s'est donnés pour "paraphraser" l'événement terrible, césure de l'histoire de son peuple. Le langage même par lequel l'artiste s'attaque à la réalité insaisissable est soumis à des violences syntaxiques étonnantes, à une mutilation des significations et se trouve par suite quelque part dans un équilibre instable entre le rationnel et l'irrationnel. On mesure dès lors toute l'importance de la langue dans ce système narratif qui se tient résolument aux limites, non pas de la représentation, mais du représentable.

Narration lyrique, dialogues, chansons, séquences oniriques s'enchaînent selon une stratégie et une technique prodigieuses, conférant au roman une architecture d'ensemble puisque ce qui compte réellement c'est le vaste remuement de tout ce qui se structure, s'organise en un. Ce dispositif textuel qui procède par collages, par fragments, nous oblige à scander le tempo de la lecture, suivre son itinéraire entrecoupé, parsemé des tourbillons, des ralentissements, des interruptions ou des silences tels qu'ils sont marqués par le vocabulaire et, également, par les jeux typographiques: italiques, espaces blancs, guillemets, ou points de suspension. La complexité de l'événement semble réclamer la nécessité de découper l'arabesque narrative en chapitres, sans titre, sans nombre, distincts, constituant une multiplicité de tableaux dont chacun pourrait être facilement ôté de 
l'ensemble, et former en soi une entité.

Un tour d'horizon est suffisant pour observer qu'il s'agit d'un texte de prose singulière: un rythme perpétuel, mais changeant, imprègne le discours tandis que les répétitions, les rappels ont un effet de rime ou de refrain. Toutefois, la virtuosité, car il y a un véritable savoir-faire, ne nous permet pas de nous installer dans la délectation esthétique: on est toujours sollicité, intrigué, afin de comprendre le message de l'auteur et à la fois d'embrasser le tempo extrêmement rapide, parfois déroutant, de son écriture brisée.

Au-delà de cet aspect, il y a l'essentiel: l'effort irrépressible de l'artiste pour nous faire croire son histoire, faire toucher, passer de la vue et de la lecture du texte au touché puisque ce qui est réel est aussi tangible. Dib nous fait toucher par les mots et non de la main, et c'est ici la dimension capitale de son oeuvre, le côté poétique. Les mots ne sont pas les mots habituels du langage articulé; inventés ou non, leurs associations inouïes qui ne rentrent pas dans le rythme ordinaire, choquent et déplacent nos images toutes faites, nos habitudes de lire. Rendre la monstruosité de la guerre à son paroxysme, c'est justement toucher à l'irreprésentable, et cela n'est réalisable que si se trouve mis en oeuvre un large éventail de moyens artistiques contradictoires lesquels exagérés, déformés choquent violemment le consommateur d'art. L'implique en actant émotionnel. Alors là, où la représentation défaille, le présent des affects supplée, et re-présente, toujours de nouveau à l'imaginaire l'inimaginable. Représente à l'imaginaire ses limites.

Notes

1 Jean Déjeux, "Romans algériens et guerre de libération". L'Esprit créateur, no. 1/1986, p. 73.

2 Jean-François Lyotard, "Discussion ou phraser après Auschwitz". Les fins de l'homme. Actes du colloque de Cerisy. Paris: Galilée, 1981, pp. 283-287.

${ }^{3}$ Rudolf Arnheim, The Genesis of a Painting. Picasso's Guernica. Berkeley: University of California Press, 1962, p. 24.

${ }^{4}$ Toutes ces dernières citations se retrouvent dans la postface au roman de Mohammed Dib, Qui se souvient de la mer. Paris: Seuil, 1962, pp. 189 - 191. Par la suite les chiffres entre parenthèses indiquent la page dans le livre cité.

${ }^{5}$ On le rappelle, c'est Apollinaire qui forge ce néologisme par lequel il désigne une façon de traduire la réalité.

${ }^{6}$ Paul Ricoeur, Le conflit des interprétations. Paris: Seuil, 1969, p. 146.

${ }^{7}$ Paul Ricoeur, ibid., p. 47. 
${ }^{8}$ Frantz Fanon, Les damnés de la terre. Paris: Maspero, 1961, p. 43.

9 Jean Louis Ferrier, De Picasso à Guernica - Généalogie d'un tableau. Paris: Denoël, 1985, p. 166.

${ }^{10}$ Mircea Eliade, Patterns in Comparative Religion. New York: New American Library, 1974, p. 411.

11 Georges Bachelard, cité par J. Déjeux, Littérature maghrébine de langue française. Sherbrooke: Naaman, 1978, p. 162.

${ }^{12}$ L'expression de Claude Ollier est citée par Abdelkebir Khatibi, Figures de l'étranger dans la littérature française. Paris: Denoël, 1987, p. 117.

${ }^{13}$ Gilles Deleuze et Felix Guattari opposent l'espace lisse du nomade (nomos) à l'espace strié de la 1'Etat (polis). Mille plateaux. Paris: Minuit, 1980, p. 479.

14 Jacques Derrida, "Donner la mort". L'éthique du don. Actes du colloque de Royaumont 1990. Paris: Métailié-Transition, 1992, p. 60.

${ }^{15}$ Hassan El-Nauty, "Roman et révolution". Présence francophone. No. 2/1971, pp. 142152.

${ }^{16}$ Jacques Derrida, op. cit., p. 9. 